\title{
Constraining dark matter capture and annihilation cross sections by searching for neutrino signature from the Earth core
}

\author{
Fei-Fan Lee ${ }^{1}$, Guey-Lin Lin $^{1}$, and Yue-Lin Sming Tsai ${ }^{2}$ \\ ${ }^{1}$ Institute of Physics, National Chiao-Tung University, Hsinchu 30010, Taiwan and \\ ${ }^{2}$ National Centre for Nuclear Research, Hoża 69, 00-681 Warsaw, Poland
}

(Dated: September 3, 2018)

\begin{abstract}
We study the sensitivity of IceCube/DeepCore detector to dark matter annihilations in the Earth core. We focus on annihilation modes $\chi \chi \rightarrow \nu \bar{\nu}, \tau^{+} \tau^{-}, b \bar{b}$, and $W^{+} W^{-}$. Both track and cascade events are considered in our analysis. By fixing the dark matter annihilation cross section $\langle\sigma v\rangle$ at some nominal values, we study the sensitivity of IceCube/DeepCore detector to dark matter spin-independent cross section $\sigma_{p}^{\mathrm{SI}}$ for $m_{\chi}$ ranging from few tens of GeV to $10 \mathrm{TeV}$. This sensitivity is compared with the existing IceCube 79-string constraint on the same cross section, which was obtained by searching for dark matter annihilations in the Sun. We compare this sensitivity to dark matter direct detection results as well, in particular the XENON100 (2012) limit and the parameter regions preferred by DAMA and CRESST-II experiments. We also present IceCube/DeepCore sensitivity to $\langle\sigma v\rangle$ as a function of $m_{\chi}$ by fixing $\sigma_{p}^{\mathrm{SI}}$ at XENON100 (2012) and XENON1T limits, respectively. This sensitivity is compared with the preferred dark matter parameter range derived from the combined fitting to PAMELA and AMS02 positron fraction data. We conclude that the search for dark matter annihilations in the Earth core provides competitive constraints on $\sigma_{p}^{\mathrm{SI}}$ and $\langle\sigma v\rangle$ in the case of low-mass dark matter. Particularly, the expected constraint on $\sigma_{p}^{\text {SI }}$ for 5 years of data taking in IceCube/DeepCore is more stringent than the current IceCube 79-string limit mentioned above.
\end{abstract}

PACS numbers: $14.60 . \mathrm{Pq}, 14.60 . \mathrm{St}$ 


\section{INTRODUCTION}

Evidences for the dark matter (DM) are provided by many astrophysical observations, although the nature of DM is yet to be uncovered. The most popular candidates for DM are weak interacting massive particles (WIMP), which we shall assume in this work. DM can be detected either directly or indirectly with the former observing the nucleus recoil as DM interacts with the target nuclei in the detector and the latter detecting final state particles resulting from DM annihilations or decays. The direct detection is possible because that the dark matter particles constantly bombard the Earth as the Earth sweeps through the local halos. As just stated, the direct detection experiments record the nuclei recoil energy of nuclei-WIMPs scattering. At present, DAMA [1], CoGeNT [2], and CRESST [3] have reported the detection of DM signal with the DM mass $m_{\chi}$ ranging from few GeV to 50 $\mathrm{GeV}$ and the spin-independent scattering cross section $\sigma_{p}^{\mathrm{SI}} \sim 10^{-4} \mathrm{pb}$. On the other hand, XENON100 [4] only collects 2 events which are consist with the background. This result then sets the limit $\sigma_{p}^{\mathrm{SI}}<2 \times 10^{-9} \mathrm{pb}$ for $m_{\chi}=55 \mathrm{GeV}$. Interestingly, recent CDMS II result [5] reports three signal events which gives a p-value $0.19 \%$ (less than $4 \sigma$ ). The corresponding best-fit values of DM parameters are $m_{\chi}=8.6 \mathrm{GeV}$ and $\sigma_{p}^{\mathrm{SI}} \sim 1.9 \times 10^{-5} \mathrm{pb}$.

DM can also be detected indirectly by measuring the positron signals from Milky Way. PAMELA observed a rise of the cosmic ray positron fraction for positron energy greater than $10 \mathrm{GeV}$ [6]. This anomalous enhancement are confirmed by Fermi-LAT [7] and the recently released AMS02 first result [8]. In the recent AMS02 result, this continuous rise on positron fraction is extended up to positron energy $\sim 350 \mathrm{GeV}$. Such a spectral behavior makes the DM annihilation explanation of the data difficult because it requires a large boost factor for annihilation cross section $\langle\sigma v\rangle$ provided the thermal equilibrium for DM in the early universe is reached with $\langle\sigma v\rangle \sim 3 \times 10^{-26} \mathrm{~cm}^{3} \mathrm{~s}^{-1}$. For example, several groups [9 15] fit the updated galactic positron fraction with AMS02 new result included. They have found that the favored DM parameter region is located at $m_{\chi} \sim$ few $\mathrm{TeV}$ and $10^{-23} \lesssim\langle\sigma v\rangle / \mathrm{cm}^{3} \mathrm{~s}^{-1} \lesssim 10^{-21}$ if DM annihilation channel $\chi \chi \rightarrow \tau^{+} \tau^{-}$is responsible for the positron excess. The favored DM mass range can be lowered to few hundred $\mathrm{GeV}$ if nearly pulsar sources are considered together with $\chi \chi \rightarrow \tau^{+} \tau^{-}$annihilations [12]. However the favored values for $\langle\sigma v\rangle$ do not decrease much in such a combined fitting. It is important to note that DM annihilations in the galactic halo are constrained by Fermi-LAT gamma ray observations [16]. The constraint 
on $\left\langle\sigma\left(\chi \chi \rightarrow \tau^{+} \tau^{-}\right) v\right\rangle$ is in fact located in the preferred DM parameter region resulting from PAMELA and AMS02 measurements for the same range of $m_{\chi}$.

It has been pointed out some time ago that the preferred DM parameter region by PAMELA and Fermi-LAT measurements can be examined through the observation of neutrinos [17 19] (see also discussions in Refs. [20, 21]) by IceCube detector augmented with DeepCore array. Indeed IceCube 22 string result on searching for DM annihilations from galactic halo [22] has set the upper limit for $\left\langle\sigma\left(\chi \chi \rightarrow \tau^{+} \tau^{-}\right) v\right\rangle$ comparable to the required annihilation cross section for explaining PAMELA and Fermi-LAT data. The IceCube sensitivity on DM signature from the galactic halo is expected to improve with the data from all 86 strings analyzed. The analysis of DeepCore array data will further enhance the sensitivity in the small $m_{\chi}$ regime [23, 24] which is of interest due to direct detection results mentioned above.

It is interesting to note that the constraints on DM capture cross section and annihilation cross section $\langle\sigma v\rangle$ can be obtained from the searching for DM annihilations from the Earth core. The detection of DM induced neutrino signature from the Earth core has been discussed previously [25-28]. It has been shown that the chemical composition of the Earth core results in several DM annihilation peaks for $m_{\chi}$ ranging from $20 \mathrm{GeV}$ to $60 \mathrm{GeV}$. These peaks do not appear for annihilations inside the Sun. Furthermore, DM annihilation rate inside the Sun is completely determined by the capture cross section (dominated by spin dependent component proportional to $\sigma_{p}^{\mathrm{SD}}$ ) while DM annihilation rate in the Earth core depends on both $\sigma_{p}^{\mathrm{SI}}$ (contribution proportional to $\sigma_{p}^{\mathrm{SD}}$ is negligible) and $\langle\sigma v\rangle$. This is understood by the fact that DM density in the former case has already reached equilibrium while DM density in the latter case has not. Hence the search for neutrino signature from the Earth core can probe both cross sections.

Model-independent sensitivity studies on IceCube detection of DM induced neutrino signature from the Earth core were reported in [29, 30] for DM mass around TeV. In this work, we consider an extended DM mass range from few tens of $\mathrm{GeV}$ to $\mathrm{TeV}$. In the low mass range, our results can be compared with direct detection results from DAMA, CoGeNT, CRESST-II and XENON100 mentioned above. In the high mass range around TeV, our results can be compared with those from cosmic ray observations by PAMELA, Fermi-LAT and AMS02. We study both muon track events and cascade events induced by neutrinos. We consider annihilation channels $\chi \chi \rightarrow \nu \bar{\nu}, \chi \chi \rightarrow \tau^{+} \tau^{-}, W^{+} W^{-}, b \bar{b}$ for signature 
neutrino productions. For non-monochromatic modes, we note that $\chi \chi \rightarrow \tau^{+} \tau^{-}$produces hardest neutrino spectrum while $\chi \chi \rightarrow b \bar{b}$ produces the softest one. We also include $\nu_{\mu} \rightarrow \nu_{\tau}$ oscillations for lower energy neutrinos.

This paper is organized as follows. In Sec. II, we discuss the neutrino flux produced in the Earth core by DM annihilations. The procedure for calculating such a flux is outlined. In Sec. III, we discuss the track and shower event rates resulting from DM annihilations in the Earth core. The background event rates from atmospheric neutrino flux are also calculated. We adopt the effective areas published by IceCube observatory for event rate calculations. In Section IV, we present IceCube/DeepCore 5-year sensitivities for detecting DM induced neutrino signature from the Earth core. We first fix the DM mass at two representative values, $m_{\chi}=50 \mathrm{GeV}$ and $m_{\chi}=2 \mathrm{TeV}$. The IceCube/DeepCore $2 \sigma$ sensitivity for 5 -year data taking is then presented as a curve on $\left(\langle\sigma v\rangle, \sigma_{p}^{\mathrm{SI}}\right)$ plane. Next, we fix the annihilation cross section $\langle\sigma v\rangle$ at conservative values, $3 \times 10^{-26} \mathrm{~cm}^{3} \mathrm{~s}^{-1}$ and $3 \times 10^{-27} \mathrm{~cm}^{3} \mathrm{~s}^{-1}$. We then present IceCube/DeepCore sensitivities to spin-independent cross section $\sigma_{p}^{\mathrm{SI}}$ as a function of $m_{\chi}$ for different assumptions on dominant DM annihilation channels. Such sensitivities are then compared with existing constraints from direct detection experiments and that obtained from the IceCube/DeepCore search of DM annihilations in the Sun. Finally, we take different experimental bounds on $\sigma_{p}^{\mathrm{SI}}$ as inputs to obtain different IceCube/DeepCore sensitivities to DM annihilation cross section on the $\left(m_{\chi}, \sigma_{p}^{\mathrm{SI}}\right)$ plane for different annihilation channels. There are thus three scenarios for the input $\sigma_{p}^{\mathrm{SI}}$ : (A) $\sigma_{p}^{\mathrm{SI}}$ favored by DAMA and CRESST-II; (B) $\sigma_{p}^{\mathrm{SI}}$ bound set by XENON100; (C) $\sigma_{p}^{\mathrm{SI}}$ bound set by XENON1T (2017) [31] assuming non-detection. We particularly compare IceCube sensitivity to $\left\langle\sigma\left(\chi \chi \rightarrow \tau^{+} \tau^{-}\right) v\right\rangle$ to the favored range on the same quantity implied by PAMELA, Fermi-LAT and AMS02. We note that neither $\chi \chi \rightarrow W^{+} W^{-}$nor $\chi \chi \rightarrow b \bar{b}$ can simultaneously fit well to PAMELA and AMS02 [12] if either channel is assumed to be dominant. We conclude in Sec. V.

\section{NEUTRINO FLUX FROM DM ANNIHILATION IN THE EARTH CORE}

To facilitate our discussions, let us define $d N_{\nu_{i}}^{f} / d E_{\nu}$ as the energy spectrum of $\nu_{i}$ produced per DM annihilation $\chi \chi \rightarrow f \bar{f}$ in the Earth core. The differential DM neutrino flux of flavor 
$i$ on the Earth surface is then given by

$$
\frac{d \Phi_{\nu_{i}}^{\mathrm{DM}}}{d E_{\nu}}=P_{\nu_{j} \rightarrow \nu_{i}}\left(E_{\nu}, D\right) \frac{\Gamma_{A}}{4 \pi D^{2}} \sum_{f} B_{\chi}^{f} \frac{d N_{\nu_{i}}^{f}}{d E_{\nu}},
$$

where $\Gamma_{A}$ is the DM annihilation rate, $B_{\chi}^{f}$ is the branching ratio for the DM annihilation channel $\chi \chi \rightarrow f \bar{f}, D$ is the distance between the source and the detector, and $P_{\nu_{j} \rightarrow \nu_{i}}\left(E_{\nu}, D\right)$ is the neutrino oscillation probability from the source to the detector.

To calculate $d \Phi_{\nu_{i}}^{\mathrm{DM}} / d E_{\nu}$, we employ WIMPSIM [32] with a total of 50000 Monte Carlo generated events. Although we are particularly interested in IceCube/DeepCore measurements, the DM neutrino flux from the Earth core is the same for all detector locations near the Earth surface due to the spherical symmetry. The oscillation probability $P_{\nu_{j} \rightarrow \nu_{i}}\left(E_{\nu}, D\right)$ is calculated with the best fit neutrino oscillation parameters summarized in Table I of Ref. [33], $\theta_{12}=33.65^{\circ}, \theta_{13}=8.93^{\circ}, \theta_{23}=38.41^{\circ}, \delta=1.08 \pi, \delta m_{21}^{2}=7.54 \times 10^{-5} \mathrm{eV}^{2}$, and $\delta m_{31}^{2}=2.47 \times 10^{-3} \mathrm{eV}^{2}$.

The DM annihilation rate $\Gamma_{A}$ can be determined by the following argument. When the Earth sweeps through DM halo, the WIMP could collide with matter inside the Earth and lose its speed. If the WIMP speed becomes less than its escape velocity, the WIMP can be captured by Earth's gravitational force and then sinks into the core of Earth. After a long peroid of accumulation, WIMPs inside the core of Earth can begin to annihilate into Standard Model particles at an appreciable rate. Among the annihilation final states, neutrino can be detected by neutrino telescopes. Let $N(t)$ be the number of WIMPs in the Earth core at time $t$, we have

$$
\frac{d N}{d t}=C_{c}-2 \Gamma_{A}(t)-C_{E} N
$$

where $C_{c}$ is the capture rate and $C_{E}$ is the evaporation rate. It has been shown that WIMPS with masses between 5-10 GeV may evaporate from the Earth [26, 28, 34, 35]. Since we are interested in the mass range $m_{\chi}>10 \mathrm{GeV}$, we neglect $C_{E}$ in our discussions. The capture rate $C_{c}$ depends on DM-nuclei elastic scattering cross section which contains spin-dependent component $\sigma_{p}^{\mathrm{SD}}$ and spin-independent component $\sigma_{p}^{\mathrm{SI}}$. The DM annihilation rate $\Gamma_{A}(t)$ is proportional to $N^{2}(t)$. One writes

$$
\Gamma_{A}(t)=\frac{1}{2} C_{A} N^{2}(t) .
$$


Taking into account the quasi-thermal distribution of WIMPs in the Earth core, the annihilation coefficient $C_{A}$ can be written as [36]

$$
C_{A}=\frac{\left\langle\sigma_{a} v\right\rangle}{V_{0}}\left(\frac{m_{\chi}}{20 \mathrm{GeV}}\right)^{2 / 3}
$$

where $V_{0}=2.3 \times 10^{25} \mathrm{~cm}^{3}$ for the Earth.

By solving $N(t)$ in Eq. (21), we obtain [35]

$$
\Gamma_{A}(t)=\frac{C_{c}}{2} \tanh ^{2}\left(\frac{t}{\tau_{A}}\right)
$$

where $t$ is the age of the macroscopic body, for example $t=4.5$ Gyr for Sun and Earth, while $\tau_{A}$ is the equilibration time scale, $\tau_{A}=\left(C_{c} C_{A}\right)^{-1 / 2}$. Numerically $\tanh ^{2}\left(t / \tau_{A}\right) \rightarrow 1$ for

$\frac{t}{\tau_{A}}>2$. In such a case $\Gamma_{A}(t)=C_{c} / 2$ so that the DM annihilation rate in the Earth core depends only on the capture rate $C_{c}$ and is independent of the annihilation cross section $\langle\sigma v\rangle$.

Since the heavy nuclei such as iron is abundant in the Earth core, the capture cross-section is enhanced due to its quadratic dependence on the nuclei atomic number. Therefore, the corresponding capture rate is given by [37]

$$
C_{c} \propto \frac{\rho_{0}}{\mathrm{GeV} \mathrm{cm}^{-3}} \times \frac{\mathrm{km} \mathrm{s}^{-1}}{\bar{v}} \times \frac{\mathrm{GeV}}{m_{\chi}} \times \frac{\sigma_{p}^{\mathrm{SI}}}{\mathrm{pb}} \times \sum_{A} F_{A}^{*}\left(m_{\chi}\right),
$$

with $\bar{v}$ the DM velocity dispersion, $\rho_{0}$ the local DM density, and $A$ the atomic number of chemical element in the Earth core. $F_{A}^{*}\left(m_{\chi}\right)$ is the product of various factors including the mass fraction of element $A$, the gravitational potential for element $A$, kinematic suppression factor, form factor, and a factor of reduced mass. The explicit form of $F_{A}^{*}\left(m_{\chi}\right)$ is not essential for our discussions. It can be found, for instance, in Ref. [37]. However, we like to stress that there are significant astrophysical uncertainties on the DM local density and its velocity distributions involved in $C_{c}$ [38]. In this work, we use approximate formulae given in [37], which are adopted by DarkSUSY [39].

\section{DM SIGNAL AND ATMOSPHERIC BACKGROUND EVENTS}

Neutrino telescope such as IceCube detects neutrinos by measuring muon track and cascade events, which are induced by neutrino-nucleon charged-current (CC) and neutralcurrent (NC) scatterings. We calculate neutrino event rate according to IceCube published 
neutrino effective area $A_{\text {eff }}^{i, k}\left(E_{\nu}\right)$ [40] of the full IceCube 86-string detector, where $k$ is interaction type for different neutrino flavor $i$. For example, for muon track events, $i$ is (anti-) muon neutrino and $k$ is (anti-) muon neutrino CC interaction. On the other hand, for cascade events, $i$ includes all three neutrino flavors. For (anti-) electron neutrinos and (anti-) tau neutrinos, $k$ runs over $\mathrm{CC}$ and $\mathrm{NC}$ interactions while $k$ is exclusively $\mathrm{NC}$ interaction for (anti-) muon neutrinos. The effective area accounts for the detection efficiency including the neutrino-nucleon interaction probability, the energy loss of muon from its production point to the detector, and the detector trigger, and analysis efficiency. Hence, the neutrino event rate from the Earth DM is given by

$$
N_{\text {signal }}=\int_{E^{\text {th }}}^{m_{\chi}} \sum_{i, k} \frac{d \Phi_{\nu_{i}}^{\mathrm{DM}}}{d E_{\nu}} A_{\mathrm{eff}}^{i, k}\left(E_{\nu}\right) d E_{\nu} d \Omega
$$

where $d \Phi_{\nu_{i}}^{\mathrm{DM}} / d E_{\nu}$ is the differential neutrino flux in the vicinity of detector for a given neutrino flavor $i$, which is given by Eq. (11), and the index $k$ can be either NC or CC interaction. The effective area can be defined as $A_{\text {eff }}^{i, k}\left(E_{\nu}\right) \approx \rho_{\text {ice }} N_{A} \sigma_{\nu_{i} N}^{k}\left(E_{\nu}\right) V_{\text {eff }}^{i, k}\left(E_{\nu}\right)$ [18], where $\rho_{\text {ice }}=0.9 \mathrm{~g} / \mathrm{cm}^{3}$ is the density of ice, $N_{A}=6.022 \times 10^{23} \mathrm{~g}^{-1}$ is the Avogadro number, $\sigma_{\nu_{i} N}^{k}\left(E_{\nu}\right)$ is the neutrino-nucleon cross-section, and $V_{\text {eff }}^{i, k}\left(E_{\nu}\right)$ is the effective volume of IceCube for different neutrino-nucleon interaction events. In this work, we shall take the detector threshold energy $E^{\text {th }}$ for IceCube/DeepCore as $10 \mathrm{GeV}$ and $100 \mathrm{GeV}$, respectively. To compute the rate for $\nu_{u}$ track events, we shall use the effective area (DeepCore+IceCube Trigger) given by Ref. [40].

Unlike the calculation of track event rate, which requires only one effective area, the calculation of cascade event rate requires 5 different effective areas. The cascade-event effective area given by Ref. [40] is only for $\nu_{e} \mathrm{CC}$ interaction events. Here we also adopt the effective area marked as DeepCore+IceCube Trigger for estimating the $\nu_{e}$ event rate. To obtain effective areas for other cascade events, we perform the rescaling

$$
A_{\mathrm{eff}}^{i, k}\left(E_{\nu}\right)=A_{\mathrm{eff}}^{\nu_{e}, \mathrm{CC}}\left(E_{\nu}\right) \times \frac{\sigma_{\nu_{i} N}^{k}\left(E_{\nu}\right)}{\sigma_{\nu_{e} N}^{\mathrm{CC}}\left(E_{\nu}\right)} \times \frac{V_{\mathrm{eff}}^{\nu_{e}, \mathrm{CC}}\left(\langle y\rangle \cdot E_{\nu}\right)}{V_{\mathrm{eff}}^{\nu_{e}, \mathrm{CC}}\left(E_{\nu}\right)},
$$

where $\langle y\rangle$ is the averaged fraction of neutrino energy $E_{\nu}$ converted into shower energy after a neutrino-nucleon CC or NC interaction. One has $\langle y\rangle=0.3$ for NC interactions of $\nu_{e}$ and $\nu_{\mu}$ while $\langle y\rangle=1$ for $\nu_{e} \mathrm{CC}$ interaction [41]. For $\nu_{\tau}$, the fraction $\langle y\rangle$ resulting from $\nu_{\tau} \mathrm{CC}$ interaction and subsequent tau-lepton decay is approximately $0.6 \times\left\langle y_{h}\right\rangle+0.4$ where $\left\langle y_{h}\right\rangle$ is the energy fraction of $\nu_{\tau}$ taken by hadrons in $\nu_{\tau}$-nucleon $\mathrm{CC}$ interaction [42]. The factor 
0.4 is the visible energy fraction in tau lepton decays, which can be estimated by using PYTHIA [43]. It should be noted that the final effective area/effective volume for cascade events may be significantly reduced due to the background rejection cut. In a recent IceCube 79-string result on atmospheric $\nu_{e}$ flux measurement [44], the final effective volume for $\nu_{e}$ events is much smaller than the one at the DeepCore filter level for lower energies while such a difference is within an order of magnitude for $E_{\nu} \gtrsim 10^{3} \mathrm{GeV}$, as can be seen from Fig. 2 of that paper. Since our sensitivity calculations for cascade events are based upon effective areas in Ref. [40], we only present IceCube/DeepCore sensitivities for $E^{\text {th }}=100 \mathrm{GeV}$ in the case of cascade events.

For calculating the atmospheric (ATM) background event rates, we use the atmospheric neutrino flux taken from Ref. [45]. Because the intrinsic ATM $\nu_{\tau}$ flux is negligible, we only consider ATM $\nu_{e}$ and $\nu_{\mu}$ fluxes at the production point. It is essential to include the effect of neutrino transmissions through the Earth. Since the Earth becomes opaque to neutrinos only for $E_{\nu}>40 \mathrm{TeV}$ [42], the neutrino transmission in our interested energy range is essentially the neutrino oscillation effect. Hence the ATM background event rate is given by

$$
N_{\text {background }}=\int_{E^{\text {th }}}^{E_{\mathrm{max}}} \sum_{i, k} \frac{d \Phi_{\nu_{i}}^{\mathrm{ATM}}}{d E_{\nu}} A_{\mathrm{eff}}^{i, k}\left(E_{\nu}\right) d E_{\nu} d \Omega,
$$

where $d \Phi_{\nu_{i}}^{\mathrm{ATM}} / d E_{\nu}$ is ATM neutrino flux in the vicinity of the detector. Such a flux is given by

$$
\frac{d \Phi_{\nu_{i}}^{\mathrm{ATM}}}{d E_{\nu}}=\frac{d \Phi_{0, \nu_{j}}^{\mathrm{ATM}}}{d E_{\nu}} P_{\nu_{j} \rightarrow \nu_{i}}\left(E_{\nu}, L\left(\theta_{\nu}\right)\right)
$$

where $d \Phi_{0, \nu_{j}}^{\mathrm{ATM}} / d E_{\nu}$ is the ATM neutrino flux at the source, $P_{\nu_{j} \rightarrow \nu_{i}}\left(E_{\nu}, L\left(\theta_{\nu}\right)\right)$ is the neutrino oscillation probability with $L\left(\theta_{\nu}\right)$ the neutrino traversing distance through the Earth along the direction of $\theta_{\nu}$. For comparing with the signal event rate induced by Earth DM annihilation with DM mass $m_{\chi}, E_{\max }$ is taken as $m_{\chi}$ in Eq. (9). We remark that $E_{\max }$ should differ from $m_{\chi}$ in practice due to the energy resolution effect. However the impact of energy resolution on our sensitivity estimation will be shown insignificant in the next section.

In Fig. 1, we show the number of neutrino events in 5 years from DM annihilations $\left(\chi \chi \rightarrow \tau^{+} \tau^{-}\right)$and ATM background as functions of the maximum open angle $\psi_{\max }$ (left) and the DM mass $m_{\chi}$ (right), respectively. Here, we take $\langle\sigma v\rangle=3 \times 10^{-26} \mathrm{~cm}^{3} \mathrm{~s}^{-1}$ and $\sigma_{p}^{\mathrm{SI}}$ the 90\% CL upper limit from XENON100. From Eq. (44), we can see the annihilation coefficient $C_{A}$ is inverse proportional to a $m_{\chi}$ dependent effective volume $V_{\text {eff }}=V_{0}\left(20 \mathrm{GeV} / m_{\chi}\right)^{2 / 3}$, 

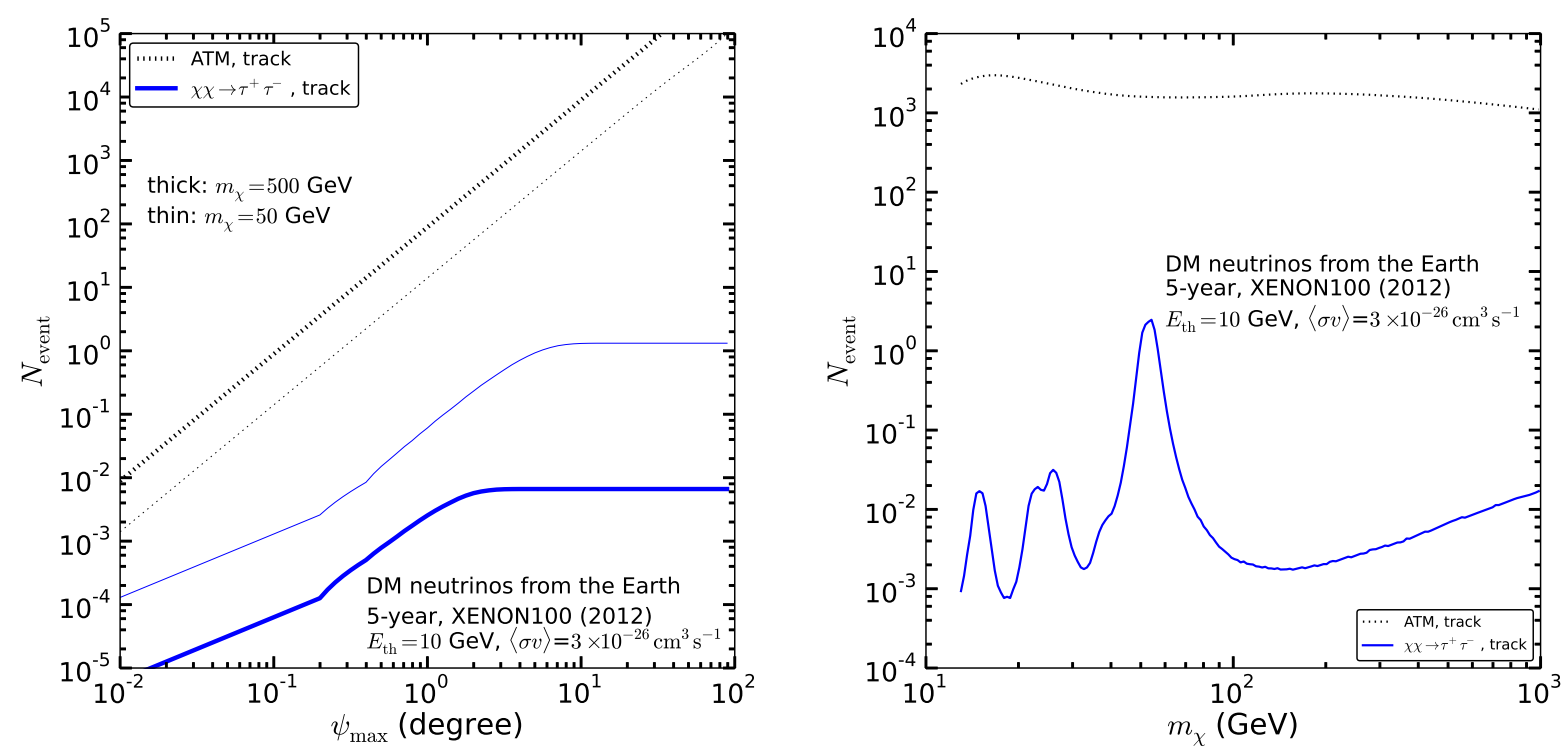

Fig. 1: The events collected in 5 year. We display the total events in the detector as function of $\psi_{\max }$ (left) and $m_{\chi}$ (right).

which describes the volume of dark matter occupation in the Earth core. Hence it is seen from the left panel of Fig. 1 that $N_{\text {events }}$ reaches to the maximum for sufficiently large $\psi_{\max }$ that can cover the entire DM populated region in the Earth core. The critical value of $\psi_{\max }$ for covering the DM populated region in the Earth core is a function of $m_{\chi}$, which we denote as $\psi_{\max }^{\mathrm{c}}\left(m_{\chi}\right)$. We have

$$
\psi_{\max }^{\mathrm{c}}\left(m_{\chi}\right)=\max \left[\sin ^{-1}\left(\frac{1}{R_{\oplus}} \times\left(\frac{3 V_{\mathrm{eff}}\left(m_{\chi}\right)}{4 \pi}\right)^{\frac{1}{3}}\right), 1^{\circ}\right],
$$

where $R_{\oplus}$ the radius of the Earth. The $1^{\circ}$ on the right hand side of the equation is to ensure a minimal open angle of $1^{\circ}$. We have seen that $V_{\text {eff }}\left(m_{\chi}\right)$ decreases as $m_{\chi}$ increases. Hence, $\psi_{\max }^{\mathrm{c}}$ for $m_{\chi}=500 \mathrm{GeV}$ is smaller than $\psi_{\max }^{\mathrm{c}}$ for $m_{\chi}=50 \mathrm{GeV}$, as can be seen from the left panel of Fig. 1. The right panel of Fig. 1 shows DM and ATM background event numbers as functions of $m_{\chi}$ where $\psi_{\max }$ for each $m_{\chi}$ is taken to be $\psi_{\max }^{\mathrm{c}}\left(m_{\chi}\right)$. The $N_{\text {events }}$ for DM signal peaks at three different values of $m_{\chi}$. This is due to the enhancement of capture rate when $m_{\chi}$ is close to the mass of any dominantly populated nuclei in the Earth core. In fact, the three peaks from small to large $m_{\chi}$ correspond to the resonant capture by oxygen, $\mathrm{Mg} / \mathrm{Si}$, and $\mathrm{Fe} / \mathrm{Ni}$, respectively. Effects of these resonant capture peaks have been studied in inert doublet [46] and supersymmetry neutralino DM models [47, 48]. In Ref. [49], the authors 
also included the effect from dark disc and found that the search for DM annihilation in the Earth can have the same level of sensitivity as the search for DM annihilation in the Sun for $m_{\chi} \lesssim 100 \mathrm{GeV}$.

\section{RESULT}

We present the sensitivity as a $2 \sigma$ detection significance in 5 years, calculated with the standard formula

$$
\frac{\text { DM signal }}{\sqrt{\text { ATM background }}}=2.0 \text {. }
$$

The ATM here is the number of atmospheric background events, which we calculate with the flux data from Ref. [45]. The right hand side of Eq. (12) refers to the $2 \sigma$ detection significance.

As mentioned earlier, the effect of detector energy resolution on the sensitivity estimation needs to be understood. Here we assume IceCube can determine the neutrino energy with the energy resolution $\sigma_{E} / E=50 \%$ [29]. With this energy resolution, we may compare the ATM track event rate in Eq. (9) with $E_{\max }=m_{\chi}$ and the similar event rate with $E_{\max }=3 m_{\chi} / 2$, which has taken into account the $50 \%$ energy resolution. We have found that, for $E^{\text {th }}=10 \mathrm{GeV}$ and $12<E_{\max } / \mathrm{GeV}<10^{3}$, the ratio of the ATM background event rate with $E_{\max }=3 m_{\chi} / 2$ to that with $E_{\max }=m_{\chi}$ varies between 2.7 and unity. From Eq. (12), we can see that the magnitude of DM annihilation cross section which the detector can probe is proportional to the square root of the ATM background event number. Thus the IceCube sensitivity to $\langle\sigma v\rangle$ with track events is changed only slightly by a factor $f$ in the range $1<f<1.65$. We expect similar effect for cascade events. Therefore, the effect of energy resolution on our sensitivity estimation is insignificant.

In Fig. 2, we show the 5 year sensitivity of the full IceCube 86-string detector to Earth DM signal on $\left(\langle\sigma v\rangle, \sigma_{p}^{\mathrm{SI}}\right)$ plane. We consider annihilation channels $\chi \chi \rightarrow \nu_{e} \bar{\nu}_{e}, \nu_{\mu} \bar{\nu}_{\mu}$, and $\tau^{+} \tau^{-}$. The first annihilation mode produces cascade events while the second and third channels produce both track and cascade events due to $\nu_{\mu} \rightarrow \nu_{\tau}$ oscillations. Each annihilation channel is assumed to be dominant when IceCube/DeepCore sensitivity to that channel is derived. The open angle $\psi_{\max }$ for collecting events from the Earth core is taken to be $5^{\circ}$ for both track and cascade events. This is reasonable for track events and achievable for

high energy cascade events [50] such as those produced with $m_{\chi}=2 \mathrm{TeV}$ described in the 

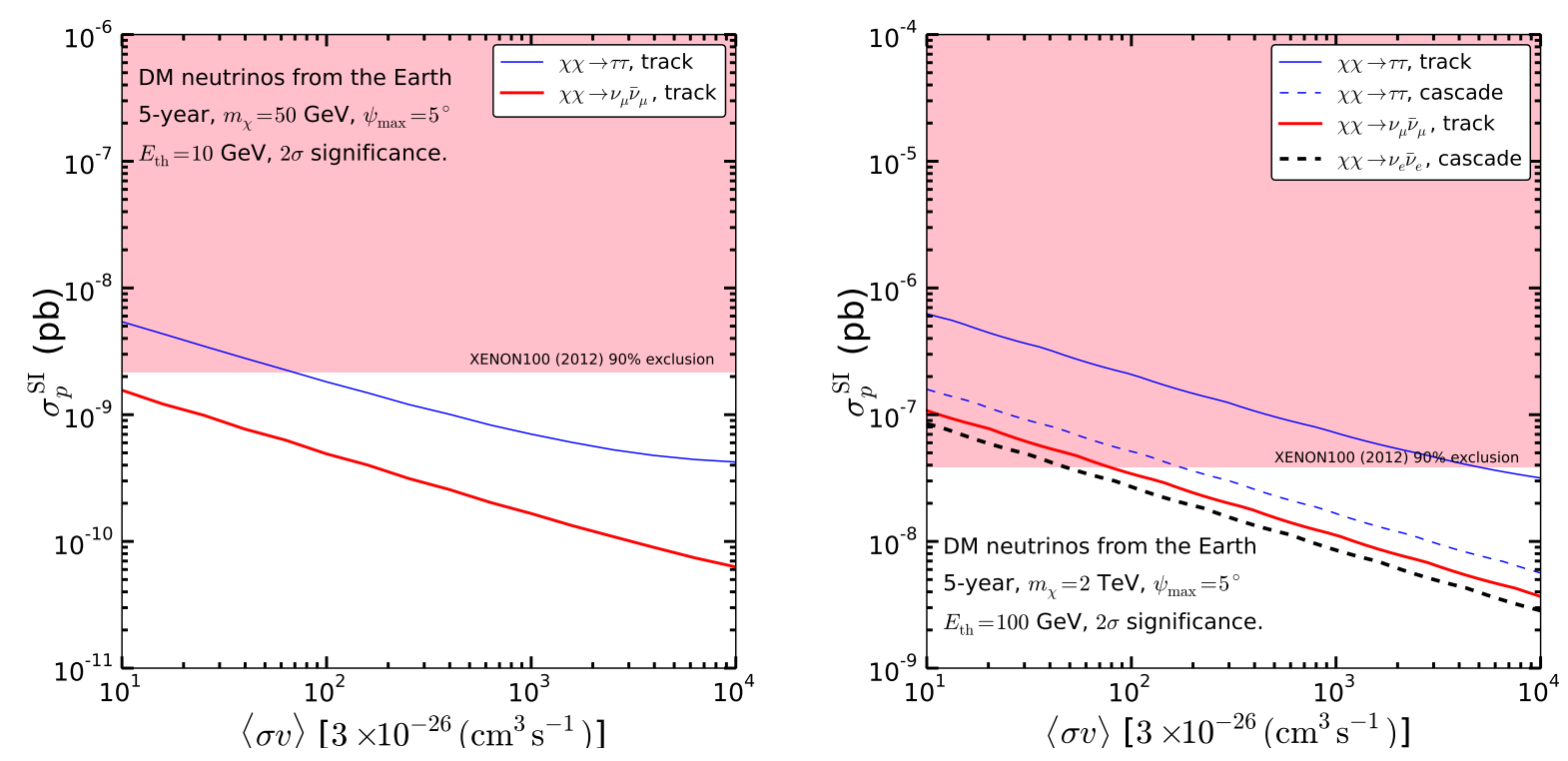

Fig. 2: The 5 year sensitivity in $2 \sigma$ significance on $\left(\langle\sigma v\rangle, \sigma_{p}^{\mathrm{SI}}\right)$ plane. In the left panel, the DM mass is $50 \mathrm{GeV}$ and the threshold energy is $10 \mathrm{GeV}$. The DM mass is $2 \mathrm{TeV}$ and threshold energy is taken to be $100 \mathrm{GeV}$ in the right panel. Solid lines are sensitivities with track events while dashed lines are sensitivities with cascade events. The pink shaded area represents the $90 \%$ exclusion from XENON100 (2012) result.

right panel. Since we only consider cascade events with $E^{\text {th }}=100 \mathrm{GeV}$, the sensitivities with cascade events are only presented in the right panel. In the right panel, we can see that $\chi \chi \rightarrow \nu_{e} \bar{\nu}_{e}$ channel is most sensitive to $\sigma_{p}^{\mathrm{SI}}$. In particular, this channel provides better sensitivity than $\chi \chi \rightarrow \nu_{\mu} \bar{\nu}_{\mu}$ channel due to relatively less ATM background events.

We note that the 5 year sensitivity curve on $\left(\langle\sigma v\rangle, \sigma_{p}^{\mathrm{SI}}\right)$ plane is almost linear in logarithmic scale so that $\sigma_{p}^{\mathrm{SI}}$ approximates to $\langle\sigma v\rangle^{-k}$ with slope $-k$. As $\langle\sigma v\rangle$ increases, a smaller scattering cross section $\sigma_{p}^{\mathrm{SI}}$ is sufficient to achieve the same detection significance. However, Eq. (3) implies that this trend cannot continue indefinitely. As $\tanh \left(\frac{t}{\tau_{A}}\right)$ is driven to the plateau by a sufficiently large $\langle\sigma v\rangle, C_{c}$ must approach to a constant value for maintaining the same annihilation rate $\Gamma_{A}$. This then implies that $\sigma_{p}^{\text {SI }}$ also approaches to a constant value. In the reverse direction where $\langle\sigma v\rangle$ decreases, a larger $\sigma_{p}^{\text {SI }}$ is required to achieve the same detection significance. On the other hand, the XENON100 limit (pink shaded region) eventually sets the upper bound for $\sigma_{p}^{\mathrm{SI}}$. This constraint is clearly seen for $\chi \chi \rightarrow \tau^{+} \tau^{-}$ channel with $m_{\chi}=2 \mathrm{TeV}$. Due to XENON100 limit, such a channel cannot produce enough 
neutrino events in IceCube for reaching $2 \sigma$ sensitivity in 5 years, unless the boost factor for $\langle\sigma v\rangle$ is larger than 1000.

It is clear that XENON100 limit sets a $m_{\chi}$-dependent bound on $\langle\sigma v\rangle$ for each annihilation channel. However, if one takes different experimental bounds on $\sigma_{p}^{\text {SI }}$, the prospect of observing neutrinos from the Earth core differs drastically. There are thus three possibilities: Case A: Neutrino observation implied by DAMA and CRESST-II favored parameter space; Case B: $\langle\sigma v\rangle$ exclusion limits implied by XENON100 bound on $\sigma_{p}^{\mathrm{SI}}$; Case C: Pessimistic scenario by assuming non-detection of XENON1T (2017).

Below we shall discuss in turn these three cases.

\section{A. Neutrino observation implied by DAMA and CRESST-II}

In Fig. 3, we present IceCube/DeepCore sensitivities to $\chi \chi \rightarrow \tau^{+} \tau^{-}, W^{+} W^{-}$, and $b \bar{b}$ annihilations in the Earth core on the $\left(m_{\chi}, \sigma_{p}^{\text {SI }}\right)$ plane for track events with different $\psi_{\max }$ and $\langle\sigma v\rangle$ with $E^{\text {th }}=10 \mathrm{GeV}$. The sensitivities to $\chi \chi \rightarrow W^{+} W^{-}$(black dashed-doted) and $\chi \chi \rightarrow \tau^{+} \tau^{-}$(grey solid) are comparable, albeit the $W^{+} W^{-}$channel only opens at $m_{\chi}>m_{W}$. The experimental upper limits of $\chi \chi \rightarrow b \bar{b}$ (red dashed-squared line) and $\chi \chi \rightarrow W^{+} W^{-} / \tau^{+} \tau^{-}$(red solid-squared line) are taken from IceCube 79 -string result on the search for muon neutrino events induced by DM annihilations in the Sun [51]. We note that the constraint on $\chi \chi \rightarrow W^{+} W^{-} / \tau^{+} \tau^{-}$stands for a constraint on $\chi \chi \rightarrow W^{+} W^{-}$for $m_{\chi}>m_{W}$ and a constraint on $\chi \chi \rightarrow \tau^{+} \tau^{-}$for $m_{\chi}<m_{W}$. For $m_{\chi} \lesssim 100 \mathrm{GeV}$, it is seen that $\sigma_{p}^{\text {SI }}$ can be better probed by detecting DM induced neutrino signature from the Earth core than that from the Sun.

The favoured region of DAMA at higher $m_{\chi}$ is not compatible with IceCube 79-string constraint on $\chi \chi \rightarrow \tau^{+} \tau^{-}$. This region can also be probed by searching for neutrinos from $\chi \chi \rightarrow \tau^{+} \tau^{-}$and $b \bar{b}$ annihilations in the Earth core. In fact, the search for muon track events induced by $\chi \chi \rightarrow \tau^{+} \tau^{-}$with $\langle\sigma v\rangle=3 \times 10^{-26} \mathrm{~cm}^{3} \mathrm{~s}^{-1}$ can probe the full allowed region of DAMA and most of the allowed region of CRESST-II. With a 10 times smaller $\langle\sigma v\rangle$, the full allowed region of DAMA can still be probed by the same annihilation channel.

From Fig. 2, we can see that the sensitivity is correlated as $\sigma_{p}^{\mathrm{SI}} \sim\langle\sigma v\rangle^{-k}$. Therefore, if one takes DAMA and CRESST-II favored regions as input, it is possible to probe $\langle\sigma v\rangle$ to a value much smaller than $3 \times 10^{-26} \mathrm{~cm}^{3} \mathrm{~s}^{-1}$ as shown by Fig. 4. In this figure, it is assumed 

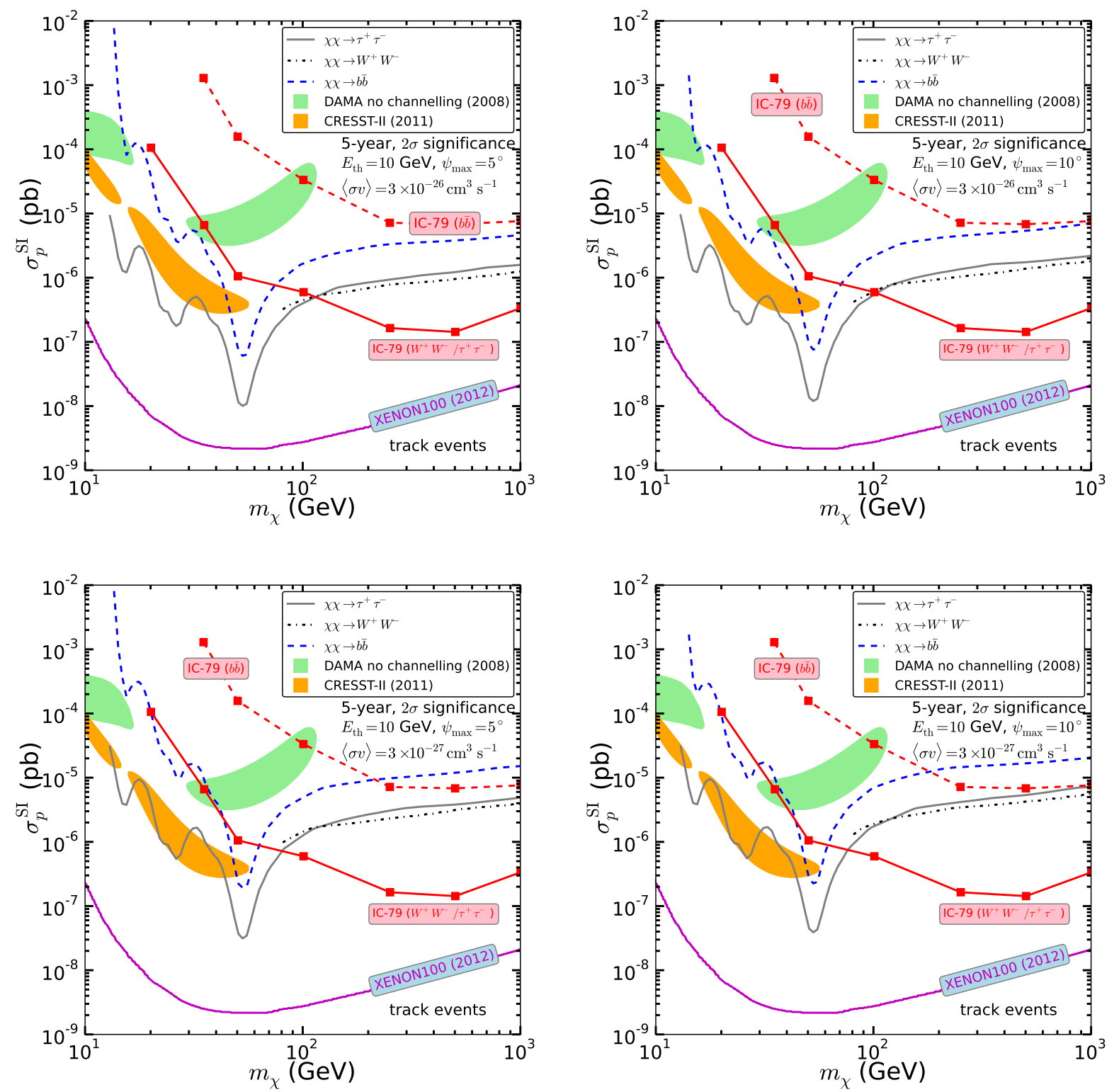

Fig. 3: The IceCube/DeepCore 5 year sensitivity curves on the $\left(m_{\chi}, \sigma_{p}^{\mathrm{SI}}\right)$ plane for $\chi \chi \rightarrow$ $\tau^{+} \tau^{-}, W^{+} W^{-}$, and $b \bar{b}$ annihilation channels. The upper figures are based on $\langle\sigma v\rangle=3 \times$ $10^{-26} \mathrm{~cm}^{3} \mathrm{~s}^{-1}$ while the lower figures are based on $\langle\sigma v\rangle=3 \times 10^{-27} \mathrm{~cm}^{3} \mathrm{~s}^{-1}$. The track-event sensitivities with $\psi_{\max }=5^{\circ}$ are presented in figures on the left column and that with $\psi_{\max }=10^{\circ}$ are presented in figures on the right column. The energy threshold is taken to be $10 \mathrm{GeV}$. The IceCube 79-string upper limits on $\chi \chi \rightarrow b \bar{b}$ (red dashed-squared line) and $\chi \chi \rightarrow W^{+} W^{-} / \tau^{+} \tau^{-}$ (red solid-squared line) from the search for DM-induced neutrino signature from the Sun are also shown for comparison [51]. 

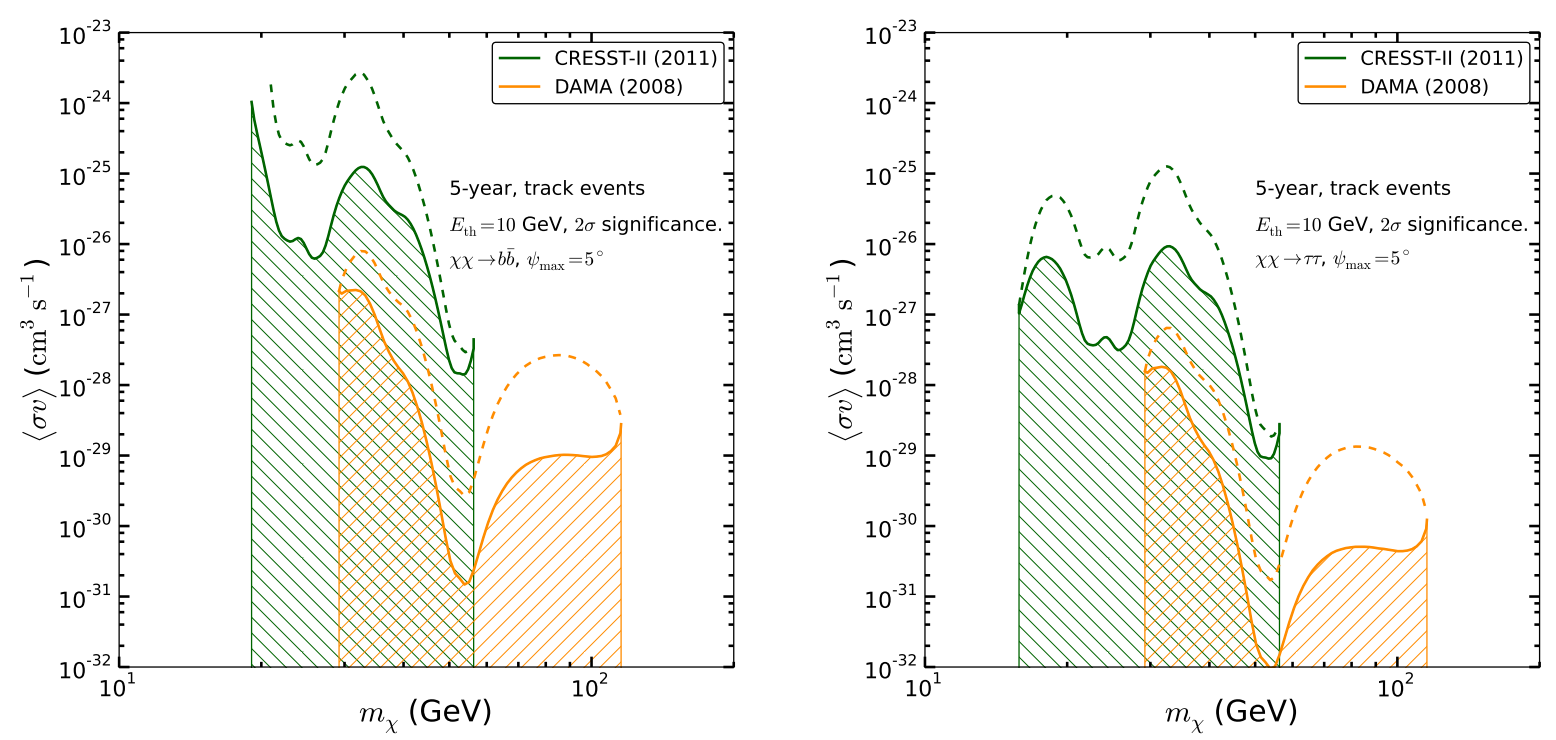

Fig. 4: The IceCube/DeepCore 5 year track-event sensitivity curves in $2 \sigma$ significance on the $\left(m_{\chi},\langle\sigma v\rangle\right)$ plane for $E_{\mathrm{th}}=10 \mathrm{GeV}$ and $\psi_{\max }=5^{\circ}$. The $\sigma_{p}^{\mathrm{SI}}$ input values are taken from no channeling DAMA and CRESST-II shown in Fig. 3. The figure in the left panel is for $\chi \chi \rightarrow b \bar{b}$ channel while the figure on the right panel is for $\chi \chi \rightarrow \tau^{+} \tau^{-}$channel. The dashed/solid lines correspond to sensitivities obtained with input $\sigma_{p}^{\text {SI }}$ given by the lower/upper boundary of DAMA and CRESST contours. The allowed regions for $\langle\sigma v\rangle$ are indicated by hatched areas.

that DM parameter regions are those given by DAMA and CRESST-II. We then present the IceCube/DeepCore 5 year track-event sensitivity curves on the $\left(m_{\chi},\langle\sigma v\rangle\right)$ plane. We take

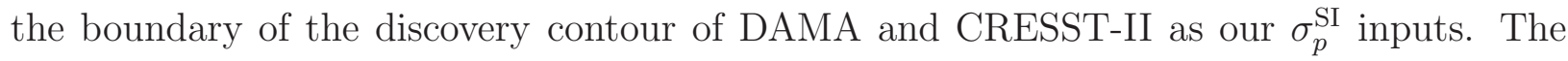
upper IceCube/DeepCore sensitivity curves (thin lines) are driven by the lower boundary of $\sigma_{p}^{\text {SI }}$ contours of DAMA and CRESST-II, while the lower sensitivity curves (thick lines) are driven by the upper boundary of $\sigma_{p}^{\mathrm{SI}}$ contours. We do not use the data of CoGent because their favoured DM mass range is below the energy threshold $E_{\text {th }}=10 \mathrm{GeV}$. For the same reason, we also ignore the $m_{\chi}<15 \mathrm{GeV}$ favoured regions of DAMA and CRESST-II. We choose the open angle $\psi_{\max }=5^{\circ}$. Because of the large capture rate $C_{c}$ resulted from the iron resonance region, $\langle\sigma v\rangle$ for this $m_{\chi}$ range can be probed to values much smaller than the thermal average cross section $\langle\sigma v\rangle \sim 3 \times 10^{-26} \mathrm{~cm}^{3} \mathrm{~s}^{-1}$. 


\section{B. $\langle\sigma v\rangle$ exclusion limits implied by XENON100 bound on $\sigma_{p}^{\mathrm{SI}}$}

We can take the XENON100 90\% upper limit as the input $\sigma_{p}^{\mathrm{SI}}$. Let us begin by taking $E^{\text {th }}=10 \mathrm{GeV}$ and consider only track events. The 5-year IceCube/DeepCore $2 \sigma$ sensitivities to $\left\langle\sigma\left(\chi \chi \rightarrow \nu_{\mu} \bar{\nu}_{\mu}\right) v\right\rangle$ and $\left\langle\sigma\left(\chi \chi \rightarrow \tau^{+} \tau^{-}\right) v\right\rangle$ as functions of $m_{\chi}$ are presented in Fig. 5. We note that the annihilation channel $\chi \chi \rightarrow \tau^{+} \tau^{-}$can give rise to track events due to the $\nu_{\tau} \rightarrow \nu_{\mu}$ oscillations for lower $E_{\nu}$. The results are obtained by considering track events with $\psi_{\max }=5^{\circ}$. The yellow shaded region corresponds to the steady state with $\tanh \left(\frac{t}{\tau_{A}}\right) \sim 1$, which is caused by a sufficiently large $\langle\sigma v\rangle$ when $\sigma_{p}^{\mathrm{SI}}$ is fixed at the current XENON100 upper limit. In this case, the number of DM trapped in the Earth core reaches to the equilibrium value since $\Gamma_{A}=C_{c} / 2$. As a result, the annihilation rate which dictates the neutrino flux is determined entirely by the capture rate and is independent of $\langle\sigma v\rangle$. The latter only determines the number of DM in the equilibrium. Hence the measurement of neutrino flux in the steady state can only determine $\sigma_{p}^{\mathrm{SI}}$.

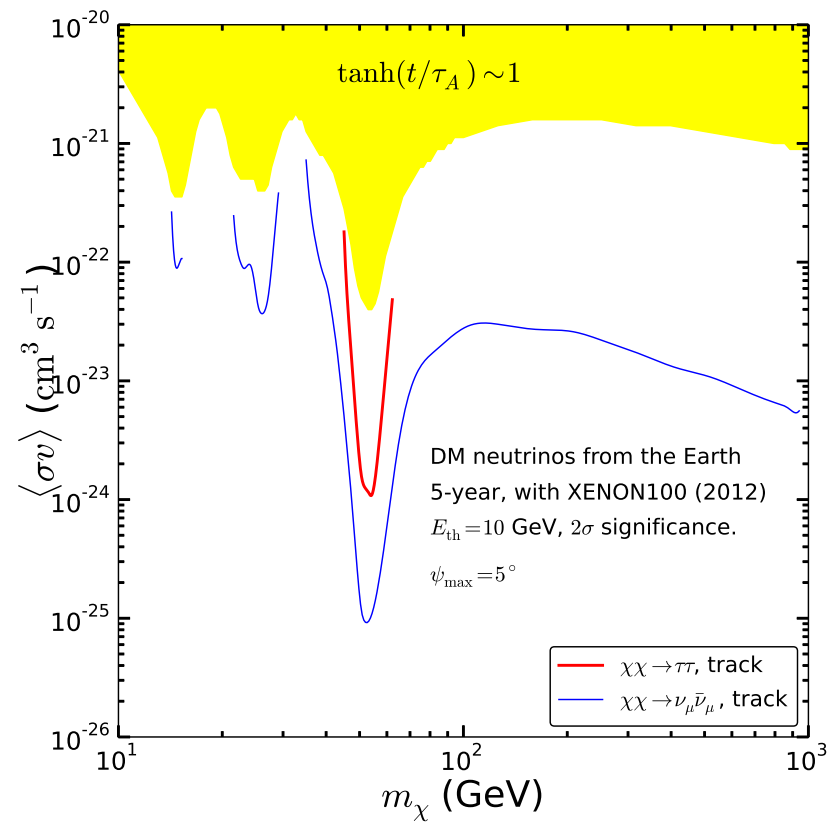

Fig. 5: The 5-year IceCube/DeepCore $2 \sigma$ sensitivities to $\left\langle\sigma\left(\chi \chi \rightarrow \nu_{\mu} \bar{\nu}_{\mu}\right) v\right\rangle$ and $\left\langle\sigma\left(\chi \chi \rightarrow \tau^{+} \tau^{-}\right) v\right\rangle$ as functions of $m_{\chi}$ with track events. The $\sigma_{p}^{\mathrm{SI}}$ is taken from XENON100 $90 \%$ upper limit. The yellow shaded area corresponds to the steady state with $\tanh \left(\frac{t}{\tau_{A}}\right) \sim 1$. We take $E^{\text {th }}=10 \mathrm{GeV}$ and $\psi_{\max }=5^{\circ}$. 
We can see the strongest limit comes from the iron resonance region $m_{\chi} \sim 50 \mathrm{GeV}$ where the capture rate $C_{c}$ peaks. On the other hand, the weakest bound of $\langle\sigma v\rangle$ occurs at the lowest point of XENON100 $\sigma_{p}^{\text {SI }}$ upper limit located at $m_{\chi} \sim 100-200 \mathrm{GeV}$. Moreover, the curves are broken near the boundary of the yellowed region. Hence, for each annihilation channel, there exists a range of $m_{\chi}$ where the $2 \sigma$ significance curve disappears. This means that the DM event number in this $m_{\chi}$ range cannot reach the $2 \sigma$ significance before $\langle\sigma v\rangle$ reaches to the steady state $\tanh \left(\frac{t}{\tau_{A}}\right) \sim 1$. One should bear in mind that this yellowed region varies with the input $\sigma_{p}^{\mathrm{SI}}$.

We next take $E^{\text {th }}=100 \mathrm{GeV}$ and consider both track and cascade events. We present in Fig. [6 the IceCube/DeepCore sensitivities to $\langle\sigma v\rangle$ of various channels as functions of $m_{\chi}$. The result in the upper left panel is obtained by taking $\psi_{\max }=\psi_{\max }^{\mathrm{c}}\left(m_{\chi}\right)$, while $\psi_{\max }$ is taken to be $5^{\circ}, 10^{\circ}$ and $50^{\circ}$ for results in the upper right, lower left and lower right panels, respectively. For $\chi \chi \rightarrow \tau^{+} \tau^{-}$channel, we also plot the $5 \sigma$ confidence region favoured by PAMELA and AMS02 positron fraction data [9] for comparison. One can see that the search for $\chi \chi \rightarrow \tau^{+} \tau^{-}$cascade events can probe almost all the $5 \sigma$ favoured region by PAMELA and AMS02 with $\psi_{\max }=5^{\circ}$. For comparison, we also estimate the IceCube/DeepCore sensitivity to the DM annihilation cross section in the galactic halo with a $100 \mathrm{GeV}$ threshold energy by using the method of Ref. [24] and the energy dependent effective volume $V_{\text {eff }}(E)$ [40]. The 5 year sensitivity upper limit of $\left\langle\sigma\left(\chi \chi \rightarrow \tau^{+} \tau^{-}\right) v\right\rangle$ obtained from searching for neutrino cascade events from the galactic center with $\psi_{\max }=50^{\circ}$ relative to the direction of galactic center is also plotted. We note that this sensitivity is independent of $\sigma_{p}^{\mathrm{SI}}$, unlike the search for DM annihilations in the Earth core. One can see that the search for galactic DM annihilations by IceCube/DeepCore can probe the entire $5 \sigma$ confidence region favoured by PAMELA and AMS02 in 5 years of running.

It is interesting to note that $\chi \chi \rightarrow \tau^{+} \tau^{-}$at the current energy range also produces track events since tau lepton can decay into muon neutrinos. In Fig. 7, we summarize the sensitivities with track events for $\tau^{+} \tau^{-}$channel with different open angles, $\psi_{\max }=1^{\circ}$ (red solid), $2^{\circ}$ (green dashed-dot), $5^{\circ}$ (black dot), $10^{\circ}$ (grey solid), and $\psi_{\max }=\psi_{\max }^{\mathrm{c}}\left(m_{\chi}\right)$ (blue dashed). We note that the sensitivity curves for $\psi_{\max }=1^{\circ}$ and $2^{\circ}$ cross at $m_{\chi} \sim 600 \mathrm{GeV}$. In other words, for $m_{\chi}<600 \mathrm{GeV}$, the numerator in Eq. (12) increases faster than the denominator as $\psi_{\max }$ increases from $1^{\circ}$ to $2^{\circ}$. (see the left panel of Fig. 1). Moreover, we note that the sensitivity to $\langle\sigma v\rangle$ can change by more than one order of magnitude as the 

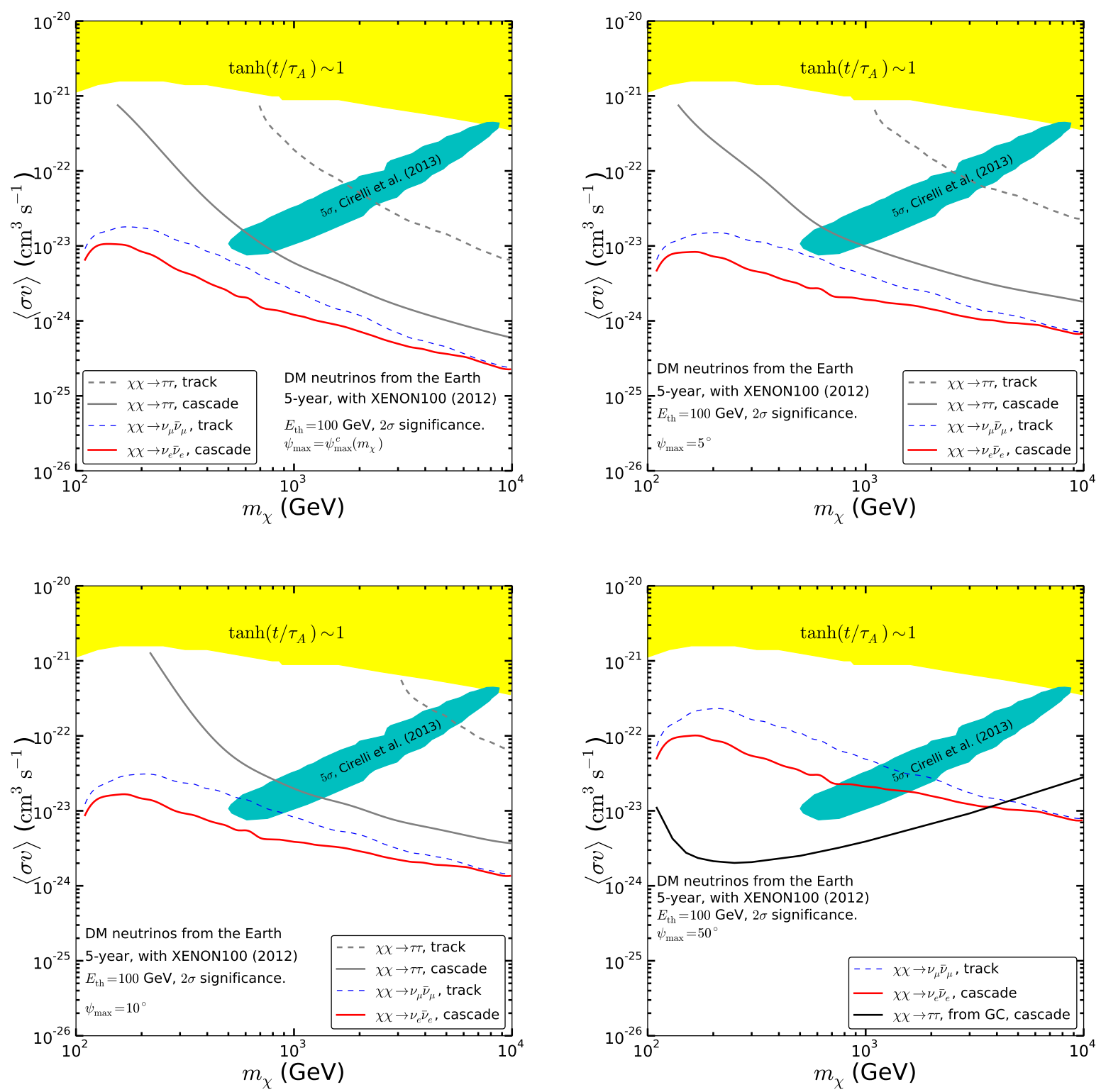

Fig. 6: The 5-year IceCube/DeepCore $2 \sigma$ sensitivities to $\langle\sigma v\rangle$ of various channels as functions of $m_{\chi}$ with track and cascade events. The threshold energy is taken to be $100 \mathrm{GeV}$ and the $m_{\chi}$ range is extended to $10 \mathrm{TeV}$. The cyan contours refer to $5 \sigma$ confidence region of PAMELA and AMS02 combined analysis, for $\chi \chi \rightarrow \tau^{+} \tau^{-}$channel, taken from Ref. [9]. The black solid line is the 5 year sensitivity upper limit of $\left\langle\sigma\left(\chi \chi \rightarrow \tau^{+} \tau^{-}\right) v\right\rangle$ in $2 \sigma$ significance obtained from the search of neutrino cascade events from the galactic center with $\psi_{\max }=50^{\circ}$ relative to the direction of galactic center.

open angle $\psi_{\max }$ increases from $1^{\circ}$ to $10^{\circ}$. Finally, with $\psi_{\max }=1^{\circ}$, IceCube 5 year data can probe the PAMELA and AMS02 positron favoured region for $m_{\chi} \gtrsim 2 \mathrm{TeV}$. 


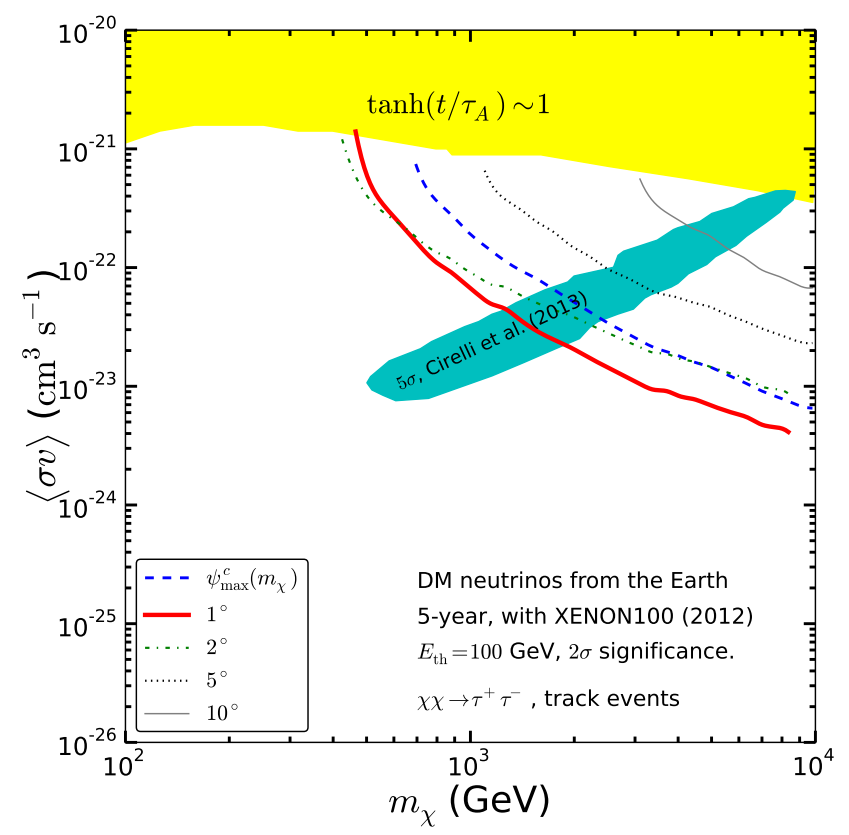

Fig. 7: The IceCube/DeepCore sensitivities to $\left\langle\sigma\left(\chi \chi \rightarrow \tau^{+} \tau^{-}\right) v\right\rangle$ with track events for several different open angles.

\section{Pessimistic scenario by assuming non-detection of XENON1T (2017)}

Finally we discuss a pessimistic scenario that DM is not detected by XENON1T (a future ton-size DM detector). In Fig. 8, instead of using the current XENON100 limit as the input for $\sigma_{p}^{\mathrm{SI}}$, we compute the IceCube 5-year $2 \sigma$ sensitivity upper limit with the projected $\sigma_{p}^{\mathrm{SI}}$ limit from XENON1T [31] as the input. We set the threshold energy at $100 \mathrm{GeV}$ and present our result for $m_{\chi}$ up to $10 \mathrm{TeV}$. Even if XENON1T $\sigma_{p}^{\mathrm{SI}}$ sensitivity limits at larger $m_{\chi}$ are weaker, only those $\langle\sigma v\rangle$ arising from monochromatic annihilation channels can be probed by IceCube, i.e., by observing cascade events from $\chi \chi \rightarrow \nu_{e} \bar{\nu}_{e}$ and $\chi \chi \rightarrow \nu_{\tau} \bar{\nu}_{\tau}$ channels (not shown on the figure), and by observing track events from $\chi \chi \rightarrow \nu_{\mu} \bar{\nu}_{\mu}$ channel. However this upper limit is not stringent since the strongest bound for $\langle\sigma v\rangle$ in this case is roughly $10^{-22}$ $\mathrm{cm}^{3} \mathrm{~s}^{-1}$. 


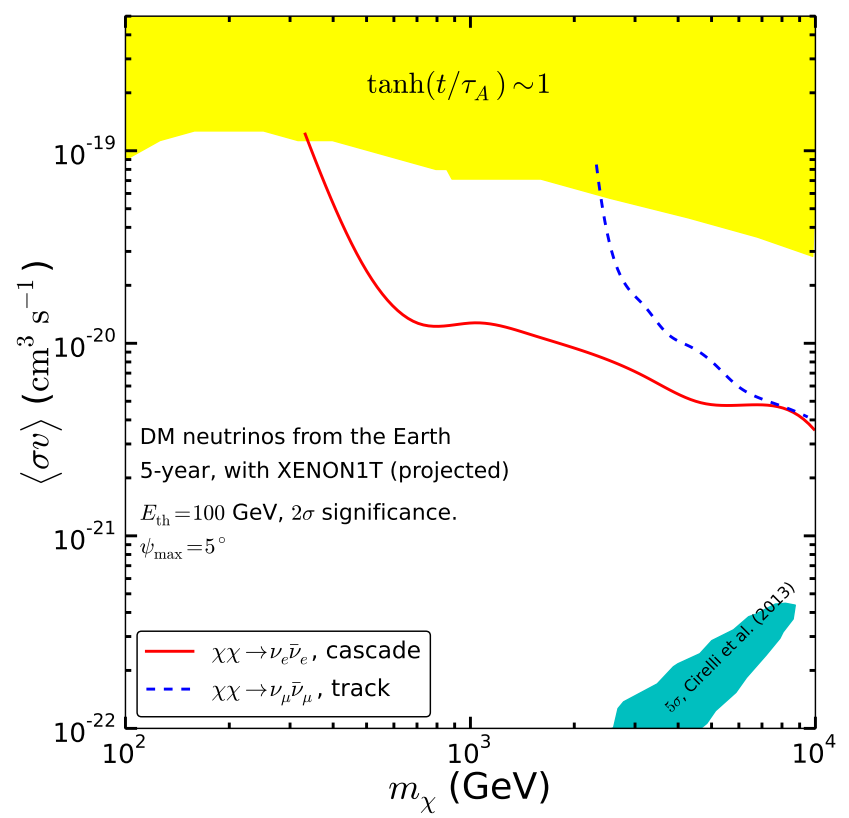

Fig. 8: The IceCube/DeepCore 5 year sensitivity with $2 \sigma$ significance on the $\left(m_{\chi},\langle\sigma v\rangle\right)$ plane. The $\sigma_{p}^{\mathrm{SI}}$ input is taken from XENON1T sensitivity curve. We take $\psi_{\max }=5^{\circ}$. The threshold energy is taken to be $100 \mathrm{GeV}$. The red solid line and blue dashed line are IceCube sensitivities to $\chi \chi \rightarrow \nu_{e} \bar{\nu}_{e}$ cascade events and $\chi \chi \rightarrow \nu_{\mu} \bar{\nu}_{\mu}$ track events, respectively.

\section{SUMMARY AND CONCLUSIONS}

In this paper, we study the neutrino signature arising from DM annihilations inside the Earth core. Applying IceCube/DeepCore effective areas, we have computed IceCube/DeepCore 5-year sensitivities in $2 \sigma$ significance with track and cascade events. From

the slope of sensitivity curves in Fig. 2, the neutrino event rate is more sensitive to $\sigma_{p}^{\mathrm{SI}}$ than $\langle\sigma v\rangle$. To illustrate the impact of $\sigma_{p}^{\mathrm{SI}}$ on the neutrino event rate, we have focused on three different scenarios according to different input $\sigma_{p}^{\mathrm{SI}}$. Hence our results can be divided by three categories.

\section{Implications from $\sigma_{p}^{\text {SI }}$ favored by of DAMA and CRESST II:}

We have compared the 5-year full IceCube/DeepCore sensitivity to $\sigma_{p}^{\text {SI }}$ derived from the search for DM annihilations in the Earth core with the recent limit on $\sigma_{p}^{\text {SI }}$ by IceCube 79string detector search for DM annihilations in the Sun. We found that the small DM mass 
region, $m_{\chi} \lesssim 100 \mathrm{GeV}$, can be better probed by detecting DM annihilations in the Earth core. By fixing $\langle\sigma v\rangle=3 \times 10^{-26} \mathrm{~cm}^{3} \mathrm{~s}^{-1}$, we found that our $\tau^{+} \tau^{-}$track event result can probe the entire DAMA allowed region and most of the CRESST-II allowed region. If one takes the large $\sigma_{p}^{\mathrm{SI}}$ favored by DAMA and CRESST-II as input, a rather low $\langle\sigma v\rangle$ is sufficient for IceCube/DeepCore to achieve $2 \sigma$ detection significance in 5 years on DM annihilations in the Earth core. It will be quite challenge for other indirect detection experiments to achieve such a sensitivity to $\langle\sigma v\rangle$ in the near future.

\section{Implication from $\sigma_{p}^{\mathrm{SI}}$ bound set by XENON100:}

We have also considered the scenario of taking the current XENON100 bound as our input $\sigma_{p}^{\mathrm{SI}}$. We have discussed the implications by taking $E^{\text {th }}$ as $10 \mathrm{GeV}$ and $100 \mathrm{GeV}$, respectively. In the former case, we study the IceCube/DeepCore sensitivities for $m_{\chi}$ up to $1 \mathrm{TeV}$ and consider only track events. In the latter case, the IceCube/DeepCore sensitivities are studied for $m_{\chi}$ up to $10 \mathrm{TeV}$ with both track and cascade events.

For $E_{\mathrm{th}}=10 \mathrm{GeV}$, we found that the strongest limit of $\langle\sigma v\rangle$ comes from the iron resonance region $m_{\chi} \sim 50 \mathrm{GeV}$. Among all the DM annihilation channels, the most stringent limit with track events arises from $\chi \chi \rightarrow \nu_{\mu} \bar{\nu}_{\mu}$ annihilation.

For $E_{\mathrm{th}}=100 \mathrm{GeV}$ where both track and cascade events are considered, the most stringent limit of $\langle\sigma v\rangle$ is from $\chi \chi \rightarrow \nu_{e} \bar{\nu}_{e}$ cascade events. Moreover, we have compared the IceCube/DeepCore sensitivities on the $\left(m_{\chi},\langle\sigma v\rangle\right)$ plane with the parameter range favored by PAMELA and AMS02 data. We found that both track and cascade events in $\chi \chi \rightarrow \tau^{+} \tau^{-}$ annihilation channel can test the PAMELA and AMS02 favored parameter space. With $\psi_{\max }=1^{\circ}$, the search for DM induced neutrino track events from the Earth core can rule out the PAMELA and AMS02 favoured parameter region at $m_{\chi} \gtrsim 2 \mathrm{TeV}$.

\section{Implication of future XENON1T sensitivity:}

We finally discussed the pessimistic scenario that DM is not discovered by the future XENON1T. With an input $\sigma_{p}^{\text {SI }}$ given by XENON1T sensitivity, we again discuss the implication on neutrino search.

With $E^{\text {th }}=100 \mathrm{GeV}$ and $10^{2}<m_{\chi} / \mathrm{GeV}<10^{4}$, we found that only those $\langle\sigma v\rangle$ arising from monochromatic annihilation channels, $\chi \chi \rightarrow \nu \bar{\nu}$, can be probed by IceCube in 5 years of data taking. However the expected bound on $\langle\sigma v\rangle$ by IceCube is disfavored by the current 
AMS-02 positron flux result.

\section{Acknowledgments}

F.F.L. is supported by the grant from Research and Development Office, National ChiaoTung University, G.L.L. is supported by National Science Council of Taiwan under Grant No. 99-2112-M-009-005-MY3 and National Center for Theoretical Sciences (NCTS), Taiwan, and Y.S.T. is funded in part by the Welcome Programme of the Foundation for Polish Science. Y.S.T. also likes to thank NCTS for hospitality during his visit.

[1] R. Bernabei et al. [DAMA and LIBRA Collaborations], Eur. Phys. J. C 67, 39 (2010).

[2] C. E. Aalseth et al. [CoGeNT Collaboration], Phys. Rev. Lett. 106, 131301 (2011) arXiv:1002.4703 [astro-ph.CO]].

[3] M. Bravin et al. [CRESST- Collaboration], Astropart. Phys. 12, 107 (1999) hep-ex/9904005.

[4] E. Aprile et al. [XENON100 Collaboration], Phys. Rev. Lett. 109, 181301 (2012) arXiv:1207.5988 [astro-ph.CO]].

[5] R. Agnese et al. [CDMS Collaboration], arXiv:1304.4279 [hep-ex]].

[6] O. Adriani et al. [PAMELA Collaboration], Phys. Rev. Lett. 106, 201101 (2011) arXiv:1103.2880 [astro-ph.HE]].

[7] M. Ackermann et al. [Fermi LAT Collaboration], Phys. Rev. D 82, 092004 (2010) arXiv:1008.3999 [astro-ph.HE]].

[8] M. Aguilar et al. [AMS Collaboration], Phys. Rev. Lett. 110, no. 14, 141102 (2013).

[9] M. Cirelli, M. Kadastik, M. Raidal and A. Strumia, Nucl. Phys. B 813, 1 (2009) arXiv:0809.2409 [hep-ph]].

[10] J. Kopp, arXiv:1304.1184 [hep-ph].

[11] A. De Simone, A. Riotto and W. Xue, arXiv:1304.1336 [hep-ph].

[12] Q. Yuan, X. -J. Bi, G. -M. Chen, Y. -Q. Guo, S. -J. Lin and X. Zhang, arXiv:1304.1482 [astro-ph.HE].

[13] I. Cholis and D. Hooper, arXiv:1304.1840 [astro-ph.HE].

[14] H. -B. Jin, Y. -L. Wu and Y. -F. Zhou, arXiv:1304.1997 [hep-ph]. 
[15] Q. Yuan and X. -J. Bi, arXiv:1304.2687 [astro-ph.HE].

[16] M. Ackermann et al. (Fermi-LAT Collaboration), Phys. Rev. D 86, 022002 (2012).

[17] D. Spolyar, M. R. Buckley, K. Freese, D. Hooper and H. Murayama, arXiv:0905.4764 [astroph.CO].

[18] M. R. Buckley, D. Spolyar, K. Freese, D. Hooper and H. Murayama, Phys. Rev. D 81, 016006 (2010).

[19] S. K. Mandal, M. R. Buckley, K. Freese, D. Spolyar and H. Murayama, Phys. Rev. D 81, 043508 (2010).

[20] L. Covi, M. Grefe, A. Ibarra and D. Tran, JCAP 1004, 017 (2010).

[21] A. E. Erkoca, M. H. Reno, and I. Sarcevic, Phys. Rev. D 82, 113006 (2010).

[22] R. Abbasi et al. [IceCube Collaboration], Phys. Rev. D 84, 022004 (2011) arXiv:1101.3349 [astro-ph.HE]].

[23] F. -F. Lee and G. -L. Lin, Phys. Rev. D 85, 023529 (2012) arXiv:1105.5719 [hep-ph]].

[24] F. -F. Lee, G. -L. Lin and Y. -L. S. Tsai, Phys. Rev. D 87, 025003 (2013) arXiv:1209.6226 [hep-ph]].

[25] K. Freese, Phys. Lett. B 167, 295 (1986).

[26] A. Gould, Astrophys. J. 321, 571 (1987).

[27] J. Lundberg and J. Edsjo, Phys. Rev. D 69, 123505 (2004) astro-ph/0401113.

[28] L. M. Krauss, M. Srednicki and F. Wilczek, Phys. Rev. D 33, 2079 (1986).

[29] I. F. M. Albuquerque, L. J. Beraldo e Silva and C. Perez de los Heros, Phys. Rev. D 85, 123539 (2012).

[30] C. Delaunay, P. J. Fox and G. Perez, JHEP 0905, 099 (2009) [arXiv:0812.3331 [hep-ph]].

[31] E. Aprile [XENON1T Collaboration], arXiv:1206.6288 [astro-ph.IM].

[32] M. Blennow, J. Edsjo and T. Ohlsson, JCAP 0801, 021 (2008) [arXiv:0709.3898 [hep-ph]].

[33] G. L. Fogli, E. Lisi, A. Marrone, D. Montanino, A. Palazzo and A. M. Rotunno, Phys. Rev. D 86, 013012 (2012) arXiv:1205.5254 [hep-ph]].

[34] M. Nauenberg, Phys. Rev. D 36, 1080 (1987).

[35] K. Griest and D. Seckel, Nucl. Phys. B 283, 681 (1987) [Erratum-ibid. B 296, 1034 (1988)].

[36] V. Berezinsky, A. Bottino, J. R. Ellis, N. Fornengo, G. Mignola and S. Scopel, Astropart. Phys. 5, 333 (1996) hep-ph/9603342.

[37] G. Jungman, M. Kamionkowski and K. Griest, Phys. Rept. 267, 195 (1996) hep-ph/9506380. 
[38] M. Cirelli, N. Fornengo, T. Montaruli, I. A. Sokalski, A. Strumia and F. Vissani, Nucl. Phys. B 727, 99 (2005) [Erratum-ibid. B 790, 338 (2008)] hep-ph/0506298].

[39] P. Gondolo, J. Edsjo, P. Ullio, L. Bergstrom, M. Schelke and E. A. Baltz, JCAP 0407, 008 (2004) astro-ph/0406204.

[40] R. Abbasi et al. [IceCube Collaboration], Astropart. Phys. 35, 615 (2012) arXiv:1109.6096 [astro-ph.IM]].

[41] J. F. Beacom and J. Candia, JCAP 0411, 009 (2004) hep-ph/0409046.

[42] R. Gandhi, C. Quigg, M. H. Reno and I. Sarcevic, Phys. Rev. D 58, 093009 (1998).

[43] T. Sjostrand, S. Mrenna and P. Z. Skands, JHEP 0605, 026 (2006) hep-ph/0603175.

[44] M. G. Aartsen et al. [IceCube Collaboration], Phys. Rev. Lett. 110, 151105 (2013) arXiv:1212.4760 [hep-ex]].

[45] M. Honda, T. Kajita, K. Kasahara, S. Midorikawa and T. Sanuki, Phys. Rev. D 75, 043006 (2007) astro-ph/0611418.

[46] S. Andreas, M. H. G. Tytgat and Q. Swillens, JCAP 0904, 004 (2009) arXiv:0901.1750 [hep-ph]].

[47] A. Bottino, F. Donato, N. Fornengo and S. Scopel, Phys. Rev. D 70, 015005 (2004) hep-ph/0401186.

[48] V. Niro, A. Bottino, N. Fornengo and S. Scopel, Phys. Rev. D 80, 095019 (2009) arXiv:0909.2348 [hep-ph]].

[49] T. Bruch, A. H. G. Peter, J. Read, L. Baudis and G. Lake, Phys. Lett. B 674, 250 (2009) arXiv:0902.4001 [astro-ph.HE]].

[50] R. Auer, Nucl. Instrum. Methods Phys. Res., Sect. A 602, 84 (2009).

[51] M. G. Aartsen et al. [IceCube Collaboration], Phys. Rev. Lett. 110, 131302 (2013) arXiv:1212.4097 [astro-ph.HE]]. 